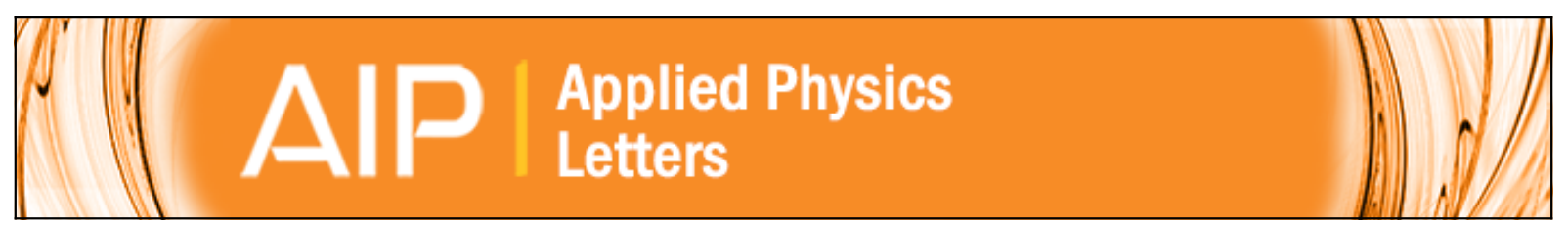

\title{
Experimental realisation of all-dielectric bianisotropic metasurfaces
}

Mikhail Odit, Polina Kapitanova, Pavel Belov, Rasoul Alaee, Carsten Rockstuhl, and Yuri S. Kivshar

Citation: Applied Physics Letters 108, 221903 (2016); doi: 10.1063/1.4953023

View online: http://dx.doi.org/10.1063/1.4953023

View Table of Contents: http://scitation.aip.org/content/aip/journal/apl/108/22?ver=pdfcov

Published by the AIP Publishing

\section{Articles you may be interested in}

All-dielectric phase-change reconfigurable metasurface

Appl. Phys. Lett. 109, 051103 (2016); 10.1063/1.4959272

Coherent perfect absorption in an all-dielectric metasurface

Appl. Phys. Lett. 108, 121901 (2016); 10.1063/1.4944635

All-dielectric metamaterial frequency selective surfaces based on high-permittivity ceramic resonators Appl. Phys. Lett. 106, 212904 (2015); 10.1063/1.4921712

Practical realization of DB metasurface

Appl. Phys. Lett. 104, 234106 (2014); 10.1063/1.4883405

Experimental verification of the concept of all-dielectric nanoantennas

Appl. Phys. Lett. 100, 201113 (2012); 10.1063/1.4719209

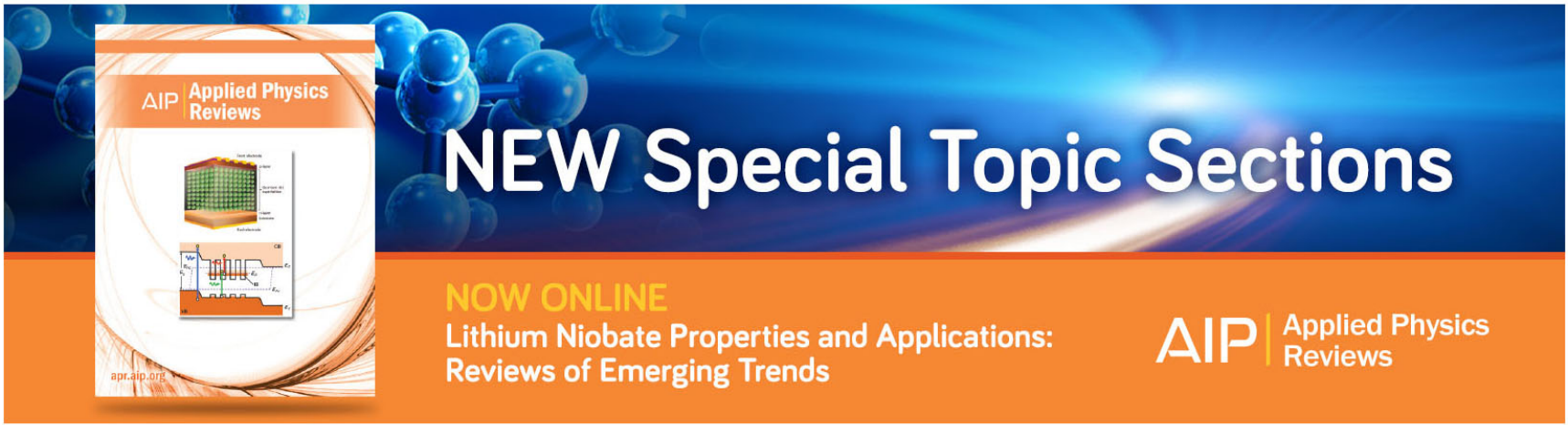




\title{
Experimental realisation of all-dielectric bianisotropic metasurfaces
}

\author{
Mikhail Odit, ${ }^{1}$ Polina Kapitanova, ${ }^{1}$ Pavel Belov, ${ }^{1}$ Rasoul Alaee,${ }^{2}$ Carsten Rockstuhl, ${ }^{2,3}$ \\ and Yuri S. Kivshar ${ }^{4,1}$ \\ ${ }^{1}$ Department of Nanophotonics and Metamaterials, ITMO University, St. Petersburg 197101, Russia \\ ${ }^{2}$ Institute of Theoretical Solid State Physics, Karlsruhe Institute of Technology, Karlsruhe 76131, Germany \\ ${ }^{3}$ Institute of Nanotechnology, Karlsruhe Institute of Technology, Karlsruhe 76021, Germany \\ ${ }^{4}$ Nonlinear Physics Centre, Australian National University, Canberra, ACT 0200, Australia
}

(Received 20 February 2016; accepted 15 May 2016; published online 31 May 2016)

\begin{abstract}
All-dielectric reciprocal metasurface based on bianisotropic scatterers operating at microwave frequencies is demonstrated experimentally. Experimental studies of a single bianisotropic particle supporting both electric and magnetic Mie-type resonances are performed, and reveal that the particle with a broken symmetry exhibits different back-scattering for the opposite excitation directions. A metasurface composed of the all-dielectric bianisotropic particles is fabricated and experimentally investigated in the frequency range of 4-9 GHz. The measured data demonstrate that the metasurface is characterized by different reflection phases when being excited from the opposite directions. At the frequency $6.8 \mathrm{GHz}$, the metasurface provides a $2 \pi$ phase change in the reflection spectrum with the amplitude close to 1. Published by AIP Publishing.

[http://dx.doi.org/10.1063/1.4953023]
\end{abstract}

Metasurfaces are two-dimensional metamaterial planar structures consisting of arrays of subwavelength particles, which allow to control and manipulate the properties of electromagnetic waves by employing the concepts and designs of flat optics. ${ }^{1}$ This includes a control of a specific phase, amplitude, and polarisation of electromagnetic waves. ${ }^{1,2}$ Metasurfaces combine many advantages of metamaterials such as their compact dimensions, broadband operation, and ability to implement various optical properties and functionalities. ${ }^{2,3}$ Many such functionalities, including perfect absorption, ${ }^{4}$ flat lensing, ${ }^{2}$ holograms, ${ }^{5}$ and surface-wave control, ${ }^{6}$ can be achieved by employing different designs of metasurfaces.

Optical metasurfaces are usually made of periodically arranged metallic particles. Recently, high-index dielectric metasurfaces attracted a lot of attention because, when compared to the plasmonic metasurfaces, they do not suffer from detrimental effects of absorption and heating. ${ }^{7-10}$ In particular, it was shown that metasurfaces with both electric and magnetic dipolar responses can suppress dramatically the wave reflection, increasing significantly their overall metasurface efficiency. ${ }^{11}$ Thus, the dielectric metasurfaces allow to control the electromagnetic wave properties in a new way by including the magnetic response of their components. ${ }^{7-9,12-14}$

A better control of electromagnetic radiation can be achieved by exploiting magnetoelectric coupling (or bianisotropy) of the metasurface elements in addition to their electric and magnetic dipolar responses. ${ }^{15-17}$ Magnetoelectric coupling occurs whenever an electric (magnetic) response is induced by a local magnetic (electric) field. ${ }^{15-17}$ This coupling leads to an asymmetric response of the metasurface when being illuminated from different directions. ${ }^{18}$ Depending on the specific kind of bianisotropy, the asymmetry may occur in reflection, transmission, or in both. ${ }^{19-21}$ There appear multiple applications associated with this effect, e.g., it is possible to polarize incident waves ${ }^{20}$ or to make a metasuface transparent from one direction while being fully opaque from the opposite direction. It has been shown experimentally that bianisotropic metasurfaces allow for extreme polarization control of light with high efficiency. ${ }^{19}$ Indeed, bianisotropy provides the most comprehensive control of an electromagnetic wave when higher-order multipole moments are absent or negligibly small in the particle response. ${ }^{17}$

Whereas bianisotropy observed with plasmonic particles is well understood by now, ${ }^{22,23}$ the observation of bianisotropy with suitably shaped high-index dielectric particles has only very recently been theoretically suggested. ${ }^{18}$ If experimentally proven, this combination would be extremely beneficial, since the low-loss advantage of dielectric based metasurfaces can be combined with the comprehensive control of the scattering response using bianisotropy. This would pave a way for a next generation of versatile, compact, and high efficiency metasurfaces.

Here, we demonstrate experimentally all-dielectric bianisotropic metasurface operating at microwave frequencies. We experimentally study the extinction spectra of a single bianisotropic high-index particle as well as transmission and reflection spectra of the bianisotropic metasurface made from such particles in the frequency range of $4-9 \mathrm{GHz}$. We experimentally demonstrate the asymmetric reflection phases for the opposite excitation directions and the possibility to achieve a $2 \pi$ phase change combined with an acceptable reflection amplitude across the entire phase spectrum of the bianisotropic all-dielectric metasurface.

The metasurface under study is composed of bianisotropic high-index dielectric particles that are periodically arranged on a square lattice with a period $a$ [Fig. 1(a)]. The individual particle has the form of a cylinder with a finite hole inside [see Fig. 1(b)]. The cylinder has a radius of $R=1 \mathrm{~cm}$ and a height of $H=1 \mathrm{~cm}$. The radius of the hole inside the cylinder is $R_{0}=0.5 \mathrm{~cm}$, and the height is $H_{0}=0.5 \mathrm{~cm}$. The dielectric material of the particle is the microwave ceramic Eccostock HiK powder with the permittivity $\varepsilon=10$ and loss tangent $\tan \delta=0.0007 .{ }^{24}$ The ambient material is air. The particle 
(a)

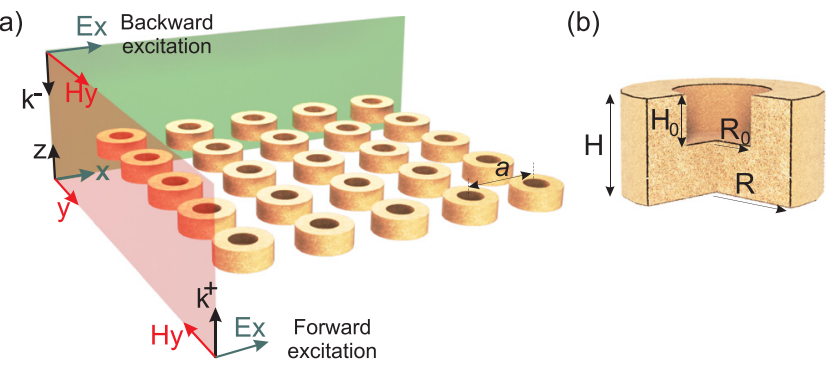

FIG. 1. (a) Schematic of a bianisotropic high-index dielectric metasurface. (b) A cutaway drawing of a single bianisotropic particle.

under consideration with broken symmetry exhibits bianisotropic properties, ${ }^{15-17}$ which were theoretically and numerically studied for optical frequencies. ${ }^{18}$

We study numerically and experimentally the microwave response of the metasurface upon plane-wave excitation. We further investigate the directional dependent response of the metasurface and distinguish between the forward and backward excitation directions. The vector triplets for the forward and backward excitation are depicted in Fig. 1(a). Under the forward excitation direction, we assume that the wavevector $k$ is co-directional with the $z$ coordinate, and refer to this propagation direction as $k^{+}$. Under the backward excitation direction, we assume that the wavevector $k$ is counter directional with the $z$ coordinate, and refer to this propagation direction as $k^{-}$.

First, we study numerically the scattering cross section of the bianisotropic particle at microwave frequencies. We distinguish forward $\left(k^{+}\right)$and backward $\left(k^{-}\right)$excitation directions. The numerical simulation was performed by using the Time Domain Solver of CST Microwave Studio. The particle surrounded with open boundaries is excited by a plane wave (normal incidence) with an electric field polarized along the $x$-axis that propagates in the $\pm z$ direction. Broadband far field monitors were defined in order to simulate the bistatic radar cross section and evaluate forward scattering (FS) and backward scattering (BS). The simulated results are shown in Fig. 2(a). As one can see, the single particle possesses an identical FS for both $\left(k^{+}\right)$and $\left(k^{-}\right)$excitation directions. However, the BS depends on the excitation direction. At the frequency $7.4 \mathrm{GHz}$, the bianisotropic particle has a minimum in the BS for the forward excitation direction $k^{+}$while for the backward excitation direction $\left(k^{-}\right)$BS is close to its maximum. The radiation patterns of the bianisotropic particle at 7.4 GHz are plotted in Fig. 2(b). There is no BS for the wave (a)

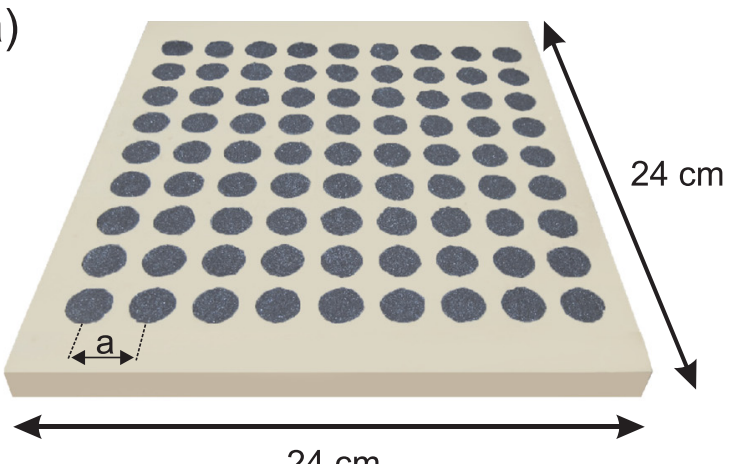

$24 \mathrm{~cm}$

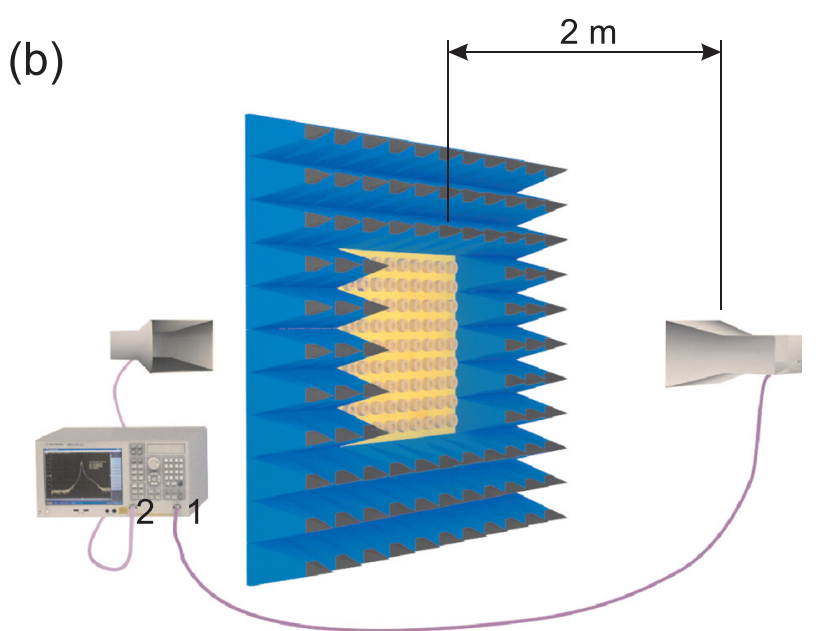

FIG. 3. (a) All-dielectric bianisotropic metasurface prototype. (b) Experimental setup for the transmission and reflection spectra measurements.

propagating in forward direction $k^{+}$. This directive radiation pattern with a zero back-scattering occurs due to the balanced condition between the induced electric and magnetic dipole moments (known as the first Kerker condition). ${ }^{12,25-27}$

To study these effects experimentally, a prototype of the bianisotropic particle has been fabricated and measured in an anechoic chamber. The inverse particle shape was drilled in a custom holder made of a Styrofoam material with a permittivity close to 1 at microwave frequencies. The holder was filled with the microwave ceramic Eccostock HiK powder. To perform a plane wave excitation and to receive the signal scattered in the forward direction, we employed a pair of identical rectangular linearly polarized wideband horn antennas (operational range $1-18 \mathrm{GHz}$ ) connected to the ports of an Agilent E8362C Vector Network Analyzer (VNA). The (a)

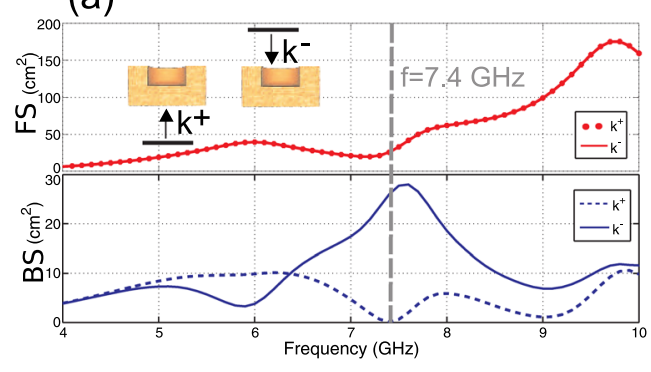

(b)

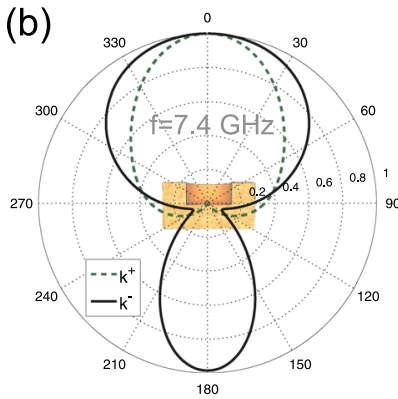

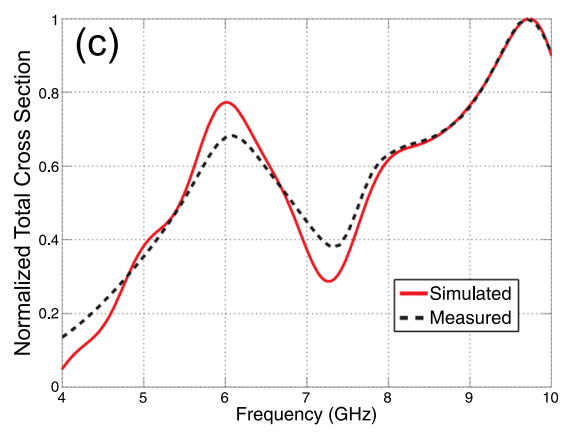

FIG. 2. (a) Simulated forward scattering (FS) and backward scattering (BS) of a bianisotropic particle. (b) Numerically simulated radiation patterns of the bianisotropic particle at the frequency $7.4 \mathrm{GHz}$. (c) Experimentally measured and numerically simulated normalized total cross section of the single particle. 


\section{Experiment}
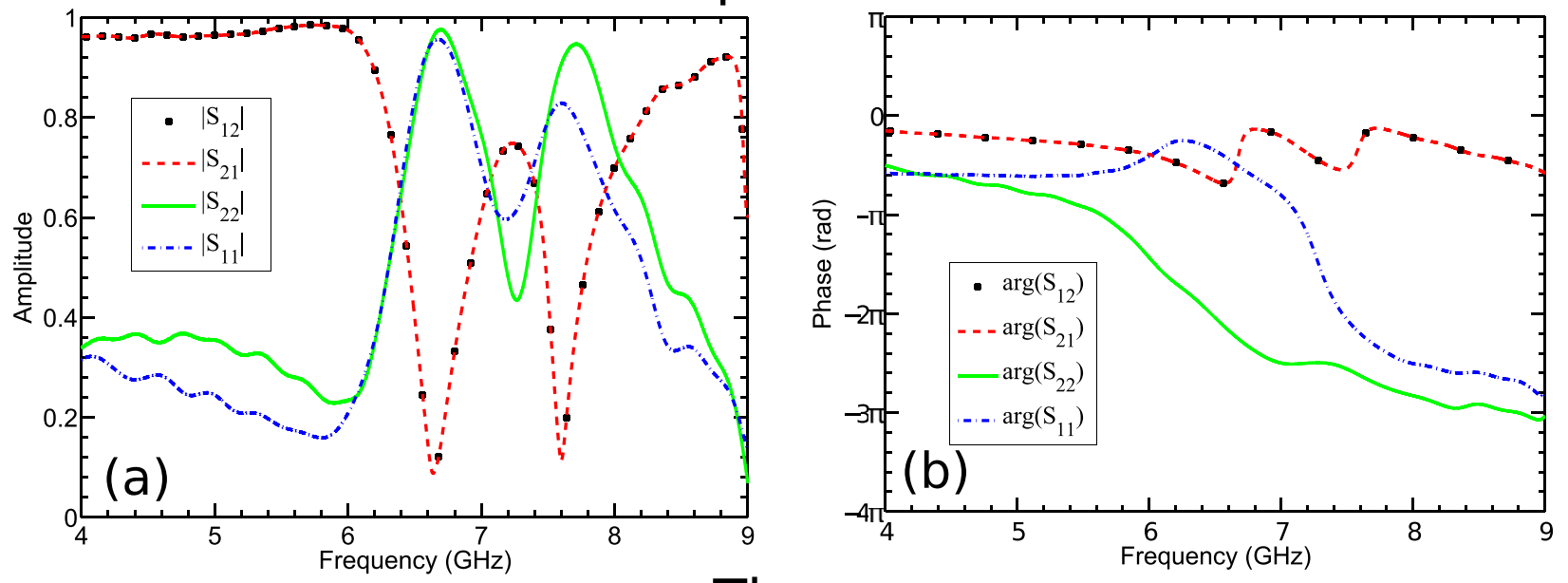

\section{Theory}
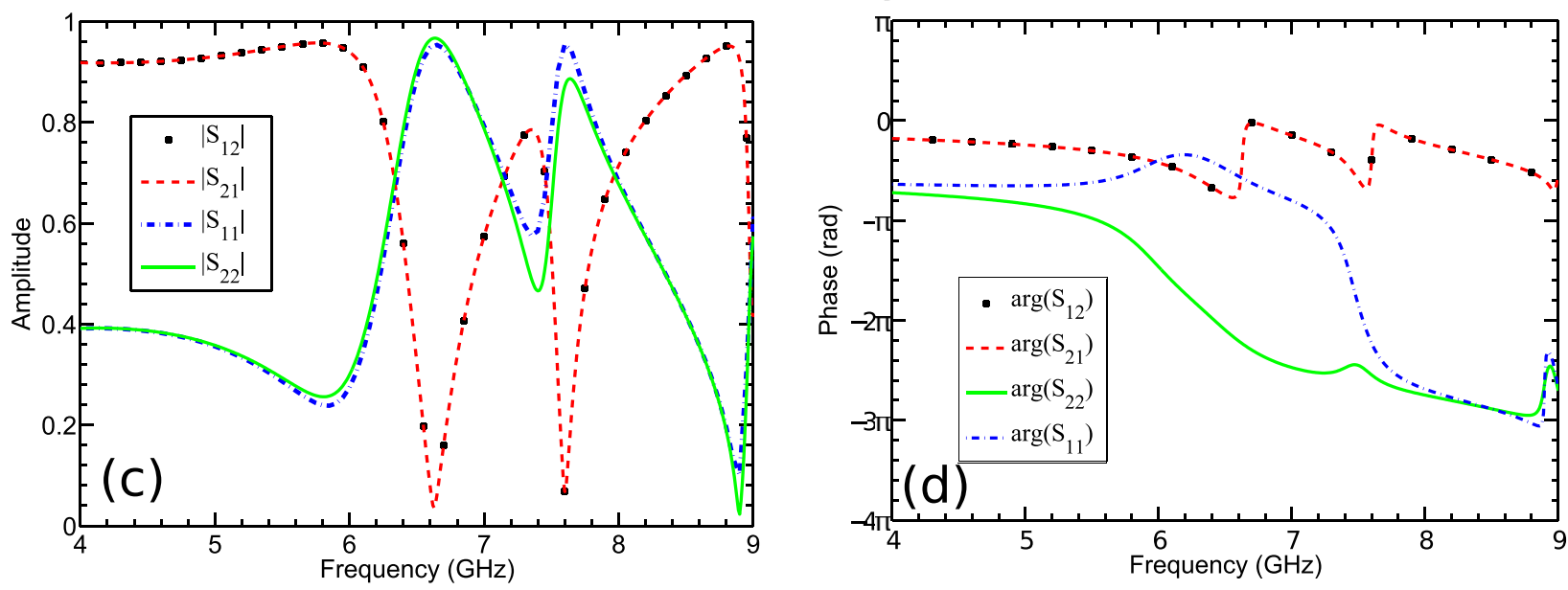

FIG. 4. (a), (b) Experimentally measured and (c), (d) numerically simulated amplitude (a), (c) and phase (b), (d) of the transmission and reflection spectra of the all-dielectric bianisotropic metasurface.

bianisotropic particle was located in the far-field at a distance of approximately $2 \mathrm{~m}$ to both the receiving and transmitting antennas. The measurement frequency range was set as $1-18 \mathrm{GHz}$. The background signal was subtracted by means of free space measurement. ${ }^{28}$ In order to minimize the effect of the additional reflections from the walls of the anechoic chamber, the measured signal was transformed into time domain with subsequent time domain gating. ${ }^{29}$ Forward scattering was then obtained from transmission coefficient. Finally, the total cross section (also known as extinction, $\sigma$ ) was extracted from the measured complex magnitude of the forward-scattered signal by means of the optical theorem. ${ }^{30}$ The experimentally measured normalized total cross section is compared with numerically simulated normalized total cross section in Fig. 2(c). We notice that the forward scattering is the same for both excitation directions since the structure is reciprocal.

Next, we fabricated and studied experimentally alldielectric bianisotropic metasurface composed of the ceramic particles studied above. An array of $9 \times 9$ inverse particle shapes was drilled in a Styrofoam material and filled with a ceramic powder. The period was $a=2.7 \mathrm{~cm}$, and the total size of the metasurface is $24 \mathrm{~cm} \times 24 \mathrm{~cm}$. A photograph of the prototype is shown in Fig. 3(a).
For experimental studies, the metasurface prototype was placed in an anechoic chamber between two horn antennas at the distance of approximately $2 \mathrm{~m}$ from each antenna. The antennas were connected to the ports of the VNA [see Fig. 3(b)]. To avoid an additional reflection from the metasurface edges and also border effects, the metasurface perimeter was surrounded by microwave absorbers. We measured the complex S-parameters of the system for the forward and backward plane wave excitations. To suppress the effects of multiple reflections between the metasurface sample and the antennas, the post-processing of measured data by means of time-domain gating was employed. ${ }^{29}$

The measured amplitude and phase of the transmission and reflection spectra are shown in Figs. 4(a) and 4(b), respectively. Figures 4(c) and 4(d) demonstrate the transmission and reflection spectra obtained from numerical simulations of the metasurface with the Frequency Domain Solver of CST Microwave Studio. In the simulations, two Bloch-Floquet ports located at the distance $1.5 \mathrm{H}$ from the metasurface were used. The periodic boundary conditions were imposed in the directions transverse to the wave incidence direction, so that the unit cell and incident wave were periodically repeated.

From the results of the numerical simulation, one can observe that the amplitude and phase of the transmission 
coefficients $S_{12}$ and $S_{21}$ are identical. This is not surprising and is indeed required for reciprocal media. The difference in the amplitude of the reflection coefficient can be caused by loss in the dielectric.

The measured amplitudes of the reflection spectra in the forward direction $\left(S_{11}\right)$ and backward direction $\left(S_{22}\right)$ are indeed different (see Fig. 4(a)), fully supported by the numerical simulation. A good agreement of the measured data and numerically simulated results is observed around the first resonance at 6-7 GHz. The difference in the amplitude of the second resonance (around $7.6 \mathrm{GHz}$ ) can be explained by the finite structure size, influence of measurement setup, and also particles' shape deviation. Note that all particles have been made from the powder. Nevertheless, the qualitative agreement is good.

The measured phases of the transmission coefficient $\mathrm{S}_{12}$ and $S_{21}$ are equally identical due to reciprocity of the system ((see Fig. 4(b)). In a sharp contrast, the measured phases of reflection coefficients $S_{11}$ and $S_{22}$ are different, which is apparently due to the presence of bianisotropy in the metasurface. At the frequency of $6.8 \mathrm{GHz}$, the measured phase difference amounts to $\arg \left(\mathrm{S}_{22}\right)-\arg \left(\mathrm{S}_{11}\right)=2 \pi$, while the measured amplitude of the reflection coefficient is close to 1 .

In conclusion, we have designed, fabricated, and studied experimentally the all-dielectric metasurface composed of high-index dielectric bianisotropic particles with different backward and forward scattering. We have demonstrated experimentally bianisotropic properties of the metasurface consisting of periodically arranged all-dielectric bianisotropic particles, which can behave as a reflect array with a $2 \pi$ reflection phase while being excited from one side, whereas remains transparent outside this operational frequency range. This feature can be essential for combining such reflectarray in multiply receiving/transmitting systems.

This work was supported by the Government of Russian Federation (Project No. GZ 3.561.2014/K), the Russian Foundation for Basic Research (Project Nos. 14-02-31761 and 15-32-20665), Grant of the President of Russian Federation MD-7841.2015.2, the German Science Foundation (Project No. RO 3640/7-1), DAAD (PPP Australien), and the Australian Research Council. The authors are thankful to A. Slobozhanyuk for providing ceramic powder.

${ }^{1}$ A. V. Kildishev, A. Boltasseva, and V. M. Shalaev, Science 339, 1232009 (2013).

${ }^{2}$ N. Yu and F. Capasso, Nat. Mater. 13, 139 (2014).

${ }^{3}$ A. F. Koenderink, A. Alù, and A. Polman, Science 348, 516 (2015).
${ }^{4}$ R. Alaee, M. Albooyeh, M. Yazdi, N. Komjani, C. Simovski, F. Lederer, and C. Rockstuhl, Phys. Rev. B 91, 115119 (2015).

${ }^{5}$ X. Ni, A. V. Kildishev, and V. M. Shalaev, Nat. Commun. 4, 2807 (2013). ${ }^{6}$ S. Sun, Q. He, S. Xiao, Q. Xu, X. Li, and L. Zhou, Nat. Mater. 11, 426 (2012).

${ }^{7}$ A. I. Kuznetsov, A. E. Miroshnichenko, Y. H. Fu, J. Zhang, and B. Lukyanchuk, Sci. Rep. 2, 492 (2012).

${ }^{8}$ A. B. Evlyukhin, S. M. Novikov, U. Zywietz, R. L. Eriksen, C. Reinhardt, S. I. Bozhevolnyi, and B. N. Chichkov, Nano Lett. 12, 3749 (2012).

${ }^{9}$ A. E. Krasnok, A. E. Miroshnichenko, P. A. Belov, and Y. S. Kivshar, Opt. Express 20, 20599 (2012).

${ }^{10}$ D. S. Filonov, A. E. Krasnok, A. P. Slobozhanyuk, P. V. Kapitanova, E. A. Nenasheva, Y. S. Kivshar, and P. A. Belov, Appl. Phys. Lett. 100, 201113 (2012).

${ }^{11}$ F. Monticone, N. M. Estakhri, and A. Alù, Phys. Rev. Lett. 110, 203903 (2013).

${ }^{12}$ I. Staude, A. E. Miroshnichenko, M. Decker, N. T. Fofang, S. Liu, E. Gonzales, J. Dominguez, T. S. Luk, D. N. Neshev, I. Brener, and Y. Kivshar, ACS Nano 7, 7824 (2013).

${ }^{13}$ U. Zywietz, A. B. Evlyukhin, C. Reinhardt, and B. N. Chichkov, Nat. Commun. 5, 3402 (2014).

${ }^{14}$ M. Decker, I. Staude, M. Falkner, J. Dominguez, D. N. Neshev, I. Brener, T. Pertsch, and Y. S. Kivshar, Adv. Opt. Mater. 3, 813 (2015).

${ }^{15}$ I. V. Lindell, A. H. Sihvola, A. J. Viitanen, and S. A. Tretyakov, Electromagnetic Waves in Chiral and Bi-Isotropic Media (Artech House, Norwood, MA, 1994), pp. 211-228.

${ }^{16}$ T. G. Mackay and W. S. Weiglhofer, "A review of homogenization studies for biaxial bianisotropic materials," in Advances in Electromagnetics of Complex Media and Metamaterials (Springer Netherlands, Dordrecht, 2002), pp. 211-228.

${ }^{17}$ A. Serdyukov, I. Semchenko, S. Tretyakov, and A. Sihvola, Electromagnetics of Bi-Anisotropic Materials: Theory and Applications (Taylor \& Francis, 2001), Vol. 11.

${ }^{18}$ R. Alaee, M. Albooyeh, A. Rahimzadegan, M. S. Mirmoosa, Y. S. Kivshar, and C. Rockstuhl, Phys. Rev. B 92, 245130 (2015).

${ }^{19}$ C. Pfeiffer, C. Zhang, V. Ray, L. J. Guo, and A. Grbic, Phys. Rev. Lett. 113, 023902 (2014).

${ }^{20}$ C. Menzel, C. Helgert, C. Rockstuhl, E.-B. Kley, A. Tünnermann, T. Pertsch, and F. Lederer, Phys. Rev. Lett. 104, 253902 (2010).

${ }^{21}$ V. S. Asadchy, Y. Ra'di, J. Vehmas, and S. A. Tretyakov, Phys. Rev. Lett. 114, 095503 (2015).

${ }^{22}$ A. V. Kildishev, J. D. Borneman, X. Ni, V. M. Shalaev, and V. P. Drachev, Proc. IEEE 99, 1691 (2011).

${ }^{23}$ A. Dimitriadis, D. Sounas, N. Kantartzis, C. Caloz, and T. Tsiboukis, IEEE Trans. Antennas Propag. 60, 5753 (2012).

${ }^{24}$ See http://www.eccosorb.com/products-eccostock-hik-powder.htm for ceramic powder datasheet.

${ }^{25}$ M. Kerker, D.-S. Wang, and C. L. Giles, J. Opt. Soc. Am. 73, 765 (1983).

${ }^{26}$ S. Person, M. Jain, Z. Lapin, J. J. Saenz, G. Wicks, and L. Novotny, Nano Lett. 13, 1806 (2013).

${ }^{27}$ R. Alaee, R. Filter, D. Lehr, F. Lederer, and C. Rockstuhl, Opt. Lett. 40, 2645 (2015)

${ }^{28}$ C. Larsson, C. Sohl, M. Gustafsson, and G. Kristensson, in Nordic Conference on Radio Science and Communications (2008), pp. 127-129.

${ }^{29}$ R. De Porrata-Doria i Yague, A. Ibars, and L. Martinez, IEEE Trans. Instrum. Meas. 47, 930 (1998).

${ }^{30}$ R. G. Newton, Am. J. Phys. 44, 639 (1976). 